\title{
Sex Hormone-Binding Globulin and Risk of Type 2 Diabetes in Women and Men
}

\author{
Eric L. Ding, Sc.D., Yiqing Song, M.D., Sc.D., JoAnn E. Manson, M.D., Dr.P.H., David J. Hunter, \\ M.B., B.S., Sc.D., Cathy C. Lee, M.D., Nader Rifai, Ph.D., Julie E. Buring, Sc.D., J. Michael \\ Gaziano, M.D., M.P.H., and Simin Liu, M.D., Sc.D. \\ From the Departments of Nutrition (E.L.D., D.J.H.) and Epidemiology (E.L.D., J.E.M., D.J.H., J.E.B.), \\ Harvard School of Public Health; the Division of Preventive Medicine (E.L.D., Y.S., J.E.M., J.E.B., \\ J.M.G.), Channing Laboratory (E.L.D., J.E.M., D.J.H.), and the Division of Aging ( J.M.G.), \\ Department of Medicine, Brigham and Women's Hospital and Harvard Medical School; the \\ Department of Laboratory Medicine, Children's Hospital and Harvard Medical School (N.R.); and \\ Massachusetts Veterans Epidemiology Research and Information Center, Veterans Affairs (VA) \\ Boston Healthcare System ( J.M.G.) - all in Boston; and VA Greater Los Angeles Healthcare \\ System (C.C.L.); the Department of Epidemiology, Program on Genomics and Nutrition and Center \\ for Metabolic Disease Prevention, UCLA School of Public Health (C.C.L., S.L.); the Department of \\ Medicine, UCLA David Geffen School of Medicine (C.C.L., S.L.); and Jonsson Comprehensive \\ Cancer Center, UCLA (S.L.) - all in Los Angeles.
}

\section{Abstract}

BACKGROUND—Circulating sex hormone-binding globulin levels are inversely associated with insulin resistance, but whether these levels can predict the risk of developing type 2 diabetes is uncertain.

\begin{abstract}
METHODS-We performed a nested case-control study of postmenopausal women in the Women's Health Study who were not using hormone therapy ( 359 with newly diagnosed type 2 diabetes and 359 controls). Plasma levels of sex hormone-binding globulin were measured; two polymorphisms of the gene encoding sex hormone-binding globulin, $S H B G$, that were robustly associated with the protein levels were genotyped and applied in mendelian randomization analyses. We then conducted a replication study in an independent cohort of men from the Physicians' Health Study II (170 with newly diagnosed type 2 diabetes and 170 controls).
\end{abstract}

RESULTS-Among women, higher plasma levels of sex hormone-binding globulin were prospectively associated with a lower risk of type 2 diabetes: multivariable odds ratios were 1.00 for the first (lowest) quartile of plasma levels, 0.16 (95\% confidence interval [CI], 0.08 to 0.33 ) for the second quartile, 0.04 (95\% CI, 0.01 to 0.12 ) for the third quartile, and 0.09 (95\% CI, 0.03 to 0.21 ) for the fourth (highest) quartile ( $\mathrm{P}<0.001$ for trend). These prospective associations were replicated among men (odds ratio for the highest quartile of plasma levels vs. the lowest quartile, 0.10; $95 \%$ CI, 0.03 to 0.36 ; $\mathrm{P}<0.001$ for trend). As compared with homozygotes of the respective wild-type allele, carriers of a variant allele of the $S H B G$ single-nucleotide polymorphism (SNP) rs6259 had $10 \%$ higher sex hormone-binding globulin levels $(P=0.005)$, and carriers of an rs6257 variant had $10 \%$ lower plasma levels $(\mathrm{P}=0.004)$; variants of both SNPs were also associated with a risk of type 2 diabetes in directions corresponding to their associated sex hormone-binding globulin levels. In mendelian randomization analyses, the predicted odds ratio of type 2 diabetes per standard-deviation

Address reprint requests to Dr. Liu at the Center for Metabolic Disease Prevention, UCLA, CHS 73-265, Box 951772, 650 Charles E. Young Dr., Los Angeles, CA 90095-1772, or at siminliu@ucla.edu.

Drs. Ding and Song contributed equally to this article. 
increase in the plasma level of sex hormone-binding globulin was 0.28 (95\% CI, 0.13 to 0.58 ) among women and 0.29 ( $95 \% \mathrm{CI}, 0.15$ to 0.58 ) among men, a finding that suggests that sex hormone-binding globulin may have a causal role in the risk of type 2 diabetes.

CONCLUSIONS-Low circulating levels of sex hormone-binding globulin are a strong predictor of the risk of type 2 diabetes in women and men. The clinical usefulness of both $S H B G$ genotypes and plasma levels in stratification and intervention for the risk of type 2 diabetes warrants further examination.

Studies since the mid-1990s have suggested that sex hormone-binding globulin may have biologic functions beyond simply regulation of the levels of free sex hormones. ${ }^{1-3}$ Classically, the primary function of sex hormone-binding globulin was thought to be the binding of circulating hormones in order to affect the bio-available fraction and sequester circulating androgens and estrogens, in particular, from biologic action. However, emerging experimental evidence indicates that even sex hormones bound to sex hormone-binding globulin may directly mediate cell-surface signaling, cellular delivery, and biologic action of sex hormones. ${ }^{1-5}$ Moreover, clinical studies have associated low circulating levels of sex hormone-binding globulin with impaired glucose control, ${ }^{6-9}$ implicating the globulin in the maintenance of glucose homeostasis. In addition, strong associations, recently reported, between plasma levels of sex hormones and the risk of type 2 diabetes show associations of similar magnitude for free sex hormones and total sex hormones, ${ }^{10}$ further indicating the bioactivity of both free and bound fractions. However, long-term prospective studies examining the role of sex hormonebinding globulin in the development of type 2 diabetes remain limited, particularly among women. ${ }^{6}$

Previous studies indicate that genetic variation may influence circulating levels of sex hormone-binding globulin. ${ }^{1-14}$ Several polymorphisms in the human sex hormone-binding globulin $(S H B G)$ gene have been found to be associated with circulating levels of sex hormonebinding globulin, ${ }^{15-19}$ insulin resistance, ${ }^{20}$ and other sex hormone-dependent conditions such as reduced bone mineral density, ${ }^{19}$ breast cancer, ${ }^{4,21}$ and prostate cancer. ${ }^{22}$ In particular, the exon 8 single-nucleotide polymorphism (SNP) rs6259, encoding an amino acid substitution of asparagine for aspartic acid at position $356(\mathrm{D} 356 \mathrm{~N}),{ }^{22}$ has been associated with increased plasma levels of sex hormone-binding globulin. ${ }^{16,22}$ However, prospective data examining $S H B G$ polymorphisms and the risk of type 2 diabetes are lacking.

We investigated the relations of plasma levels of sex hormone-binding globulin and $S H B G$ polymorphisms with the risk of type 2 diabetes in a prospective study of postmenopausal women. If $S H B G$ germ-line variants were found to be predictive of both the risk of type 2 diabetes and the plasma level of sex hormone-binding globulin, we planned to further evaluate the association between plasma levels of sex hormone-binding globulin and the risk of type 2 diabetes by using mendelian randomization with $S H B G$ genotypes as instruments to minimize residual confounding and reverse causation (since genotypes are thought to be independent of confounders and not to be modified by disease processes). (The relevant principles, assumptions, and specific methods of the mendelian randomization approach are detailed in the Supplementary Appendix, available with the full text of this article at NEJM.org.) To confirm relations between $S H B G$ genotypes, plasma levels of sex hormone-binding globulin, and risk of type 2 diabetes, we conducted replication analyses in an independent cohort of men.

\section{METHODS STUDY POPULATION}

The Women's Health Study, begun in 1993, is a randomized, double-blind, placebo-controlled, 2-by-2 factorial study of low-dose aspirin and vitamin $\mathrm{E}$ for the primary prevention of 
cardiovascular disease and cancer in 39,876 female health professionals in the United States who, at enrollment, were 45 years of age or older and did not have diabetes, cancer (other than nonmelanoma skin cancer), or cardiovascular disease. ${ }^{23}$ A total of 28,345 women provided blood samples at baseline; 12,304 were postmenopausal and were not using hormonereplacement therapy at the time of blood collection. These 12,304 women were included in our study because hormone-replacement therapy influences plasma levels of sex hormones and sex hormone-binding globulin. During a 10-year follow-up period, we identified 366 cases of newly diagnosed type 2 diabetes. Using risk-set sampling, ${ }^{24}$ we randomly selected controls from among women who remained free from type 2 diabetes and matched them to case patients, in a 1:1 ratio, according to age (within 1 year), duration of the follow-up period (within 1 month), self-reported race, and fasting status at the time of blood draw (with $72 \%$ of patients fasting, defined as there having been at least 10 hours since the previous meal). On the basis of these criteria, 359 case patients and 359 matched controls were selected from the Women's Health Study cohort.

A replication study was conducted within the Physicians' Health Study II of men ${ }^{25}$ (for details, see the Supplementary Appendix). Of the 14,641 Physicians' Health Study II participants, 11,130 provided blood samples at baseline. During 8 years of follow-up, diabetes developed in 170 initially healthy men. Risk-set sampling identical to that used in the Women's Health Study was applied to prospectively selected controls from the cohort person-time. Controls were randomly selected to match cases, in a 1:1 ratio, according to age (within 1 year), duration of the follow-up period (within 1 month), self-reported race, and time of blood draw. On the basis of these criteria, 170 cases and 170 controls were selected.

All participants in the Women's Health Study and the Physicians' Health Study II provided written informed consent before enrollment. This study was approved by the research review boards of Partners HealthCare and the University of California at Los Angeles (UCLA).

\section{LABORATORY PROCEDURES}

Plasma samples were stored in liquid nitrogen tanks until analysis. Matched case and control specimens were handled identically and were assayed in random order within each pair in the same analytical run for each cohort. Laboratory personnel were unaware of the case-control status during all assays. Plasma levels of sex hormone-binding globulin were measured with the use of a chemiluminescent immunoassay (with an Elecsys 2010 autoanalyzer, Roche Diagnostics), validated for plasma sex hormone-binding globulin. ${ }^{26}$ The coefficient of variation for sex hormone-binding globulin data among blinded quality-control samples was $2.8 \%$. Genotyping of $S H B G$ polymorphisms of women was conducted at the Harvard Cancer Center's High-Throughput Polymorphism Detection Core laboratory. Replication genotyping in men was conducted through the Program on Genomics and Nutrition at UCLA. Detailed methods of SNP selection and genotyping are described in the Supplementary Appendix. Overall, five SNPs were genotyped in women (with a success rate of $\geq 95 \%$ ). Three noninformative SNPs were excluded from analysis: the rs6260 and rs9282845 loci had a minorallele frequency of $0 \%$, and rs 6258 had a minor-allele frequency of less than $1 \%$. Two informative SNPs, rs6257 and rs6259, were included in our study and were included for genotyping in the replication study (with a success rate of $>99 \%$ ). The frequencies of the genotypes were found to be consistent with Hardy-Weinberg equilibrium among controls ( $\mathrm{P}>0.05$ for all comparisons).

\section{STATISTICAL ANALYSIS}

Baseline characteristics were compared between case patients and controls using mixed-effects regression analysis for clustered data and conditional logistic-regression analysis. We divided the distributions of plasma levels of sex hormone-binding globulin among controls into 
quartiles and compared baseline characteristics across the quartiles. Because the incidencedensity sampling method was used to match controls to case patients on the basis of the cohort person-time ${ }^{24}$ odds ratios (and $95 \%$ confidence intervals) for type 2 diabetes were computed by means of conditional logistic-regression analysis. Trend tests were computed to study the relations across increasing quartiles. In the primary multivariable model, we adjusted for the factors used in matching controls and case patients, as well as the body-mass index (BMI, treated as a continuous variable); smoking status; alcohol consumption; degree of exercise; presence or absence of family history of diabetes, history of hypertension, and past hormonereplacement therapy; years of oral contraceptive use and multivitamin use; years since menopause; and cause of menopause (see the Supplementary Appendix). To assess confounding by reproductive and sociologic covariates in a sensitivity analysis, we fit an expanded model to further adjust for age at menarche, total number of pregnancies, number of pregnancies lasting 6 months or more, age at first pregnancy of 6 months' gestation or more, marital status, and educational level. Similar analyses were carried out in men, with adjustment for BMI, smoking status, alcohol consumption, degree of exercise, systolic blood pressure, current use or nonuse of multivitamins, and presence or absence of family history of diabetes. Further sensitivity analyses excluded data for case patients in whom type 2 diabetes developed during the first 3 years of the follow-up period and accounted for waist circumference and baseline C-reactive protein (CRP) and glycated hemoglobin values. Owing to the apparent linearity of the observed associations and for parsimony, we expressed the odds ratio per natural-log standard-deviation increase when assessing the effect modification of BMI, past use of hormone-replacement therapy, years since menopause, and history of hypercholesterolemia or family history of diabetes. Geometric means and relative mean differences of plasma levels of sex hormone-binding globulin between genotypes were calculated using linear regression analysis. Results between the sexes were pooled in randomeffects models.

According to the mendelian law of independent assortment, genetic variants should be distributed independently and randomly with respect to other genetic variants, assuming no linkage disequilibrium or population stratification. ${ }^{27,28}$ Since environmental and lifestyle covariates were evenly distributed at baseline across $S H B G$ genotypes, we used genetic variants as randomization instruments to estimate the potential causal association between plasma levels of sex hormone-binding globulin and the risk of type 2 diabetes. As instrumental variables, these $S H B G$ variants seem to satisfy the three main criteria in mendelian randomization analysis: that the genotypes should be robustly associated with the intermediate phenotype, should not be associated with confounding factors that may bias the association between the intermediate phenotype and disease outcome, and should exert its effect on the clinical outcome only through the specific intermediate phenotype. ${ }^{29}$ If all these assumptions are satisfied, the coefficient estimates based on the use of $S H B G$ genotypes as instruments would be unconfounded. ${ }^{28,30}$ The mendelian randomization estimate was computed from the ratio of the coefficient of the association between genotype and disease to that of the association between genotype and sex hormone-binding globulin; the estimate reflects the potential causal effect of sex hormone-binding globulin levels on the risk of type 2 diabetes $^{27}$ (see the Supplementary Appendix).

We used the qvf command, with Murphy-Topel variance, for generalized linear models with instrumental variables ${ }^{31}$ to fit the data to regression models for plasma levels of sex hormonebinding globulin and logistic-regression models for type 2 diabetes using $S H B G$ germ-line variants as randomized instruments. Finally, prediction analyses were conducted with the use of receiver-operating-characteristic curves and $\mathrm{C}$ statistics to assess the relative predictive ability of sex hormone-binding globulin beyond that of traditional risk factors. All analyses were conducted using Stata software, version 9.2. 


\section{RESULTS}

As expected, women in whom type 2 diabetes developed (case patients) generally had more adverse risk profiles at baseline than those who remained free of the disease (controls) (Table 1). Cross-sectional analyses at baseline revealed that higher levels of sex hormone-binding globulin were associated with lower BMI, a lower likelihood of having a history of hypertension, and more favorable lipid-profile and CRP levels (Table 2). Elevated levels of sex hormone-binding globulin were strongly and consistently associated with a reduced risk of type 2 diabetes ( $\mathrm{P}$ for trend, <0.001) in both simple and multivariable analyses. Regardless of adjustment for a wide range of covariates or analysis through multiple sensitivity analyses, these findings did not materially change in direction or magnitude. The odds ratios of type 2 diabetes for quartiles 2, 3, and 4 (highest) of the sex hormone-binding globulin level, as compared with quartile 1 (lowest) were $1.00,0.16$ (95\% confidence interval [CI], 0.08 to 0.33 ), 0.04 (95\% CI, 0.01 to 0.12 ), and 0.09 (95\% CI, 0.03 to 0.21 ), respectively (P value for trend, $<0.001$ ) (Table 3). In our independent replication study in men, results also corroborated the strong inverse association between sex hormone-binding globulin levels and risk of type 2 diabetes (odds ratio for the highest vs. the lowest quartile, $0.10 ; 95 \% \mathrm{CI}, 0.03$ to 0.36 ); these findings remained highly robust in multiple sensitivity analyses (Table 3 ) and were consistent across subgroups (see the Supplementary Appendix).

In genotype analyses, neither the rs6257 nor the rs6259 SNP had genotype distributions deviating from Hardy-Weinberg equilibrium among controls. The pairwise linkage disequilibrium between $\mathrm{rs} 6257$ and $\mathrm{rs} 6259$ was minimal $\left(\mathrm{r}^{2}=0.13, \mathrm{P}=0.02\right)$. Although the rs6257 and rs6259 variants explained $2.2 \%$ of the variance in plasma levels of sex hormonebinding globulin, this statistic is not the only measure of instrument strength. Most importantly, carriers of an rs6257 variant allele (CC or CT) had a 10\% lower plasma level of sex hormonebinding globulin than the wild-type homozygotes $(\mathrm{TT})(\mathrm{P}=0.004)$, and carriage of a variant allele appeared to increase the risk of type 2 diabetes among both men and women. In contrast, carriers of an rs6259 variant allele (AA or AG) had a 10\% higher plasma level of sex hormonebinding globulin $(\mathrm{P}=0.005)$ and a lower risk of type 2 diabetes (Table 4$)$.

Owing to the low linkage disequilibrium between these two variant SNPs, we also conducted a joint association analysis involving stratification on the basis of the genotypes of rs6257 and rs6259, rather than a haplotype analysis. The findings indicated the independent and additive effects of rs6257 and rs6259 on plasma levels of sex hormone-binding globulin and on the risk of type 2 diabetes (Fig. 1). The presence of variant alleles of both SNPs yielded a difference of 20\% (95\% CI, 6 to 35) in plasma levels of sex hormone-binding globulin. Furthermore, consistent with differences in plasma levels of sex hormone-binding globulin, participants with the rs6257 wild-type genotype (TT) and a rs6259 variant genotype (AG or AA) (reflecting $21.4 \%$ of controls) had a lower risk of type 2 diabetes than those carrying an rs6257 variant allele (CT or CC genotype) and the rs6259 wild-type genotype (GG) (representing $15.6 \%$ of controls) (odds ratio, $0.43 ; 95 \%$ CI, 0.22 to 0.87 ) (Fig. 1). No association was observed between $S H B G$ polymorphisms and BMI in these two cohorts, indicating that the effects of the $S H B G$ gene on the risk of type 2 diabetes may be independent from the effect of BMI.

Using rs6257 and rs6259 alleles as instruments in mendelian randomization analysis, we ascertained that the predicted odds ratio of type 2 diabetes per natural-log standard-deviation increase in the plasma level of sex hormone-binding globulin was 0.28 (95\% CI, 0.13 to 0.58 ) in women and 0.29 (95\% CI, 0.15 to 0.58 ) in men (Table 5). These highly concordant estimates were virtually identical to odds ratios obtained through conventional multivariable analyses and were consistently observed in both women and men, indicating negligible residual confounding of sex hormone-binding globulin level and risk of type 2 diabetes in these two cohorts. 
We further conducted a relative receiver-operating-characteristic analysis to determine whether the plasma level of sex hormone-binding globulin could classify case patients with type 2 diabetes and controls more accurately than multiple established risk factors. Plasma sex hormone-binding globulin improved the relative prediction of type 2 diabetes in all models $(\mathrm{P}<0.001$ for all comparisons), including the base model comprising traditional risk factors, an expanded model comprising traditional risk factors plus CRP, an expanded model comprising traditional risk factors plus glycated hemoglobin, and a comprehensive model that included traditional risk factors, CRP, and glycated hemoglobin (see the Supplementary Appendix).

\section{DISCUSSION}

There are four main findings regarding sex hormone-binding globulin and the risk of type 2 diabetes from these two prospective studies. First, the risk of type 2 diabetes among participants with sex hormone-binding globulin levels in the highest quartile appeared to be only one tenth the risk among those with levels in the lowest quartile. Second, the rs6257 and rs6259 SHBG polymorphisms were consistently associated with plasma levels of sex hormone-binding globulin and were predictive of risk of type 2 diabetes in directions corresponding to their effects on plasma sex hormone-binding globulin levels. Third, the strong relation between plasma levels of sex hormone-binding globulin and risk of type 2 diabetes was confirmed both in standard multivariable analyses and in mendelian randomization analyses in which suitable genetic variants were used as randomization instruments. Finally, plasma levels of the globulin appeared to have a predictive ability for the risk of type 2 diabetes beyond that of traditional risk factors, including glycated hemoglobin and CRP.

Our prospective findings for plasma levels of sex hormone-binding globulin levels and risk of type 2 diabetes are consistent with results from previous cross-sectional studies of diabetes. ${ }^{6,8}$ Sex hormone-binding globulin may play an important role in the pathogenesis of type 2 diabetes, by modulating the biologic effects of sex hormones (testosterone and estrogen) on peripheral tissues (i.e., liver, muscle, and fat). Studies suggest that sex hormones bound to sex hormone-binding globulin may also be biologically active, amplifying their signaling, endocytosis, or overall biologic actions. ${ }^{1-3}$ For example, sex hormone-binding globulin has been shown to have direct cellular antagonistic properties against estrogen ${ }^{4,32}$; interaction of sex hormone-binding globulin with the cellular estrogen receptor can trigger a biologic antiestrogen response, ${ }^{4}$ a form of mediation beyond simple hormone sequestration. Our results may provide a potential explanation of the intriguing divergent effects on the risk of diabetes, observed in two randomized trials, of transdermal estradiol (which elevates plasma glucose levels) and oral estrogen (which lowers glucose levels). ${ }^{33-35}$ In direct comparisons, transdermal estradiol does not affect sex hormone-binding globulin levels, whereas oralestrogen therapy favorably increases levels of sex hormone-binding globulin. ${ }^{36-38}$

Although reverse causation has been suggested by results of cross-sectional studies in which pre-diabetic hyperinsulinemia is thought to inhibit the production of sex hormone-binding globulin, ${ }^{39,40}$ we prospectively studied participants who were apparently healthy at baseline, thus establishing a basis for detection of a temporal relationship. Potential reverse causation from undiagnosed diabetes may be a concern in our work. However, all participants were health professionals, with more valid diagnostic information and higher screening rates than those in the general population. Moreover, exclusion of the first few years of follow-up data in both studies did not affect our results, decreasing the likelihood of reverse causation. Our findings remained robust in multiple sensitivity analyses restricted to participants with glycated hemoglobin values of less than 6\%. Overall, the strength and robustness of the associations indicate that residual confounding and reverse causation are unlikely. 
Furthermore, the identification of $S H B G$ germ-line variants affecting the risk of type 2 diabetes allowed us to use mendelian randomization, rather than just conventional multivariable methods, to account for potential biases due to residual confounding and reverse causation. The estimates of genotype-disease association appear to support the plausibility of a causal relationship between plasma levels of sex hormone-binding globulin and type 2 diabetes. Analyses of data from women and men in our study yielded consistent associations among SHBG SNPs, intermediate phenotypes, and type 2 diabetes: carriage of variant rs6257 was associated with lower levels of sex hormone-binding globulin and higher risk of type 2 diabetes, whereas carriage of rs6259 was associated with higher levels of sex hormone-binding globulin and lower risk of type 2 diabetes. Elevated levels of circulating sex hormone-binding globulin among carriers of an rs6259 variant allele warrant further functional studies; the elevation may be due to an amino acid substitution of asparagine for aspartic acid (D356N) at rs6259. This locus is an $N$-glycosylation consensus site that alters the binding of sex hormonebinding globulin to membrane receptors and other proteins and reduces its clearance from the circulation, resulting in higher plasma levels of the globulin. ${ }^{22}$ The associations found for rs6257, a SNP that flanks, and is located 17 bp upstream of, exon 2 also suggests the presence of potential key splicing or regulatory elements in that region. Since $S H B G$ genetic variants may exert their effects across carriers' lifetimes, the strong odds ratios relating plasma levels of sex hormone-binding globulin to the risk of type 2 diabetes obtained from our mendelian randomization analysis may represent the average lifetime risk attributable to sex hormonebinding globulin alone, independent of traditional risk factors.

Our study has several limitations. First, the statistical power, with fewer than 600 newly diagnosed cases in the two cohorts, may be relatively limited, especially with regard to the genetic associations observed. Nevertheless, we demonstrated that two $S H B G$ SNPs were suitable randomization instruments for elucidating the relation between plasma levels of sex hormone-binding globulin and the risk of type 2 diabetes. Although residual confounding, particularly by adiposity, is possible with conventional observational analysis of biomarkers, plasma levels of sex hormone-binding globulin were only modestly correlated with adiposity, and these results were robust even after dual adjustment for BMI and waist circumference (see the Supplementary Appendix). More importantly, these interrelationships among genotypes, plasma protein levels, and phenotypes of type 2 diabetes outcomes were consistently observed in two independent cohorts.

In conclusion, our prospective studies of postmenopausal women and men showed that higher levels of circulating sex hormone-binding globulin were strongly associated with a decreased risk of type 2 diabetes. Two germ-line variants in the $S H B G$ gene were also identified as being directly associated with both plasma levels of sex hormone-binding globulin and the risk of type 2 diabetes. These strong and consistent findings, obtained with the use of multiple analytic approaches and subgroup analyses in two independent cohorts, support the notion that sex hormone-binding globulin may play an important role in the development of type 2 diabetes at both the genomic and phenotypic levels and that sex hormone-binding globulin could be an important target in stratification for the risk of type 2 diabetes and early intervention.

\section{Supplementary Material}

Refer to Web version on PubMed Central for supplementary material.

\section{Acknowledgments}

Supported by grants from the National Institutes of Health (DK066401, to Dr. Liu; HL043851, HL080467, and CA047988, to the Women's Health Study); the Burroughs Wellcome Fund (to Dr. Liu, for development of a metabolic disease research and training program at UCLA); the National Cancer Institute (CA34944, CA40360, and CA097193, to the Physicians' Health Study II); the National Heart, Lung, and Blood Institute (HL26490 and HL34595); the 
American Diabetes Association fellowship (to Dr. Ding); the Paul and Daisy Soros fellowship (to Dr. Ding); and the National Institute of Diabetes and Digestive and Kidney Diseases (K01-DK078846, to Dr. Song).

Drs. Liu, Manson, and Ding report being listed on a provisional patent application for the use of sex hormone-binding globulin for determining risk of type 2 diabetes filed by UCLA. Dr. Liu reports receiving grant support from the General Mills Bell Institute of Health and Nutrition. No other potential conflict of interest relevant to this article was reported.

We thank the dedicated participants of the Women's Health Study and the Physicians' Health Study II, as well as Gary Bradwin, Claire Ridge, Malcolm Taylor, Frank Hu, Vadim Bubes, Brian Chen, Xuyang Lu, Elizabeth Chou, Yuko You, Stephen Horvath, and Gang Li for various logistic assistance in assay development and reviews of the statistical programming and a draft of the manuscript.

\section{References}

1. Rosner W, Hryb DJ, Khan MS, Nakhla AM, Romas NA. Sex hormone-binding globulin mediates steroid hormone signal transduction at the plasma membrane. J Steroid Biochem Mol Biol 1999;69:481-5. [PubMed: 10419028]

2. Hammes A, Andreassen TK, Spoelgen R, et al. Role of endocytosis in cellular uptake of sex steroids. Cell 2005;122:751-62. [PubMed: 16143106]

3. Porto CS, Lazari MF, Abreu LC, Bardin CW, Gunsalus GL. Receptors for androgen-binding proteins: internalization and intracellular signalling. J Steroid Biochem Mol Biol 1995;53:561-5. [PubMed: 7626510]

4. Fortunati N, Becchis M, Catalano MG, et al. Sex hormone-binding globulin, its membrane receptor, and breast cancer: a new approach to the modulation of estradiol action in neoplastic cells. J Steroid Biochem Mol Biol 1999;69:473-9. [PubMed: 10419027]

5. Kahn SM, Hryb DJ, Nakhla AM, Romas NA, Rosner W. Sex hormone-binding globulin is synthesized in target cells. J Endocrinol 2002;175:113-20. [PubMed: 12379495]

6. Ding EL, Song Y, Malik VS, Liu S. Sex differences of endogenous sex hormones and risk of type 2 diabetes: a systematic review and meta-analysis. JAMA 2006;295:1288-99. [PubMed: 16537739]

7. Lindstedt G, Lundberg PA, Lapidus L, Lundgren H, Bengtsson C, Björntorp P. Low sex-hormonebinding globulin concentration as independent risk factor for development of NIDDM: 12-yr followup of population study of women in Gothenburg, Sweden. Diabetes 1991;40:123-8. [PubMed: 2015967]

8. Golden SH, Dobs AS, Vaidya D, et al. Endogenous sex hormones and glucose tolerance status in postmenopausal women. J Clin Endocrinol Metab 2007;92:1289-95. [PubMed: 17244779]

9. Sutton-Tyrrell K, Wildman RP, Matthews KA, et al. Sex-hormone-binding globulin and the free androgen index are related to cardiovascular risk factors in multiethnic premenopausal and perimenopausal women enrolled in the Study of Women Across the Nation (SWAN). Circulation 2005;111:1242-9. [PubMed: 15769764]

10. Ding EL, Song Y, Manson JE, Rifai N, Buring JE, Liu S. Plasma sex steroid hormones and risk of developing type 2 diabetes in women: a prospective study. Diabetologia 2007;50:2076-84. [PubMed: 17701157]

11. Ring HZ, Lessov CN, Reed T, et al. Heritability of plasma sex hormones and hormone binding globulin in adult male twins. J Clin Endocrinol Metab 2005;90:3653-8. [PubMed: 15755867]

12. An P, Rice T, Gagnon J, et al. A genetic study of sex hormone-binding globulin measured before and after a 20-week endurance exercise training program: the HERITAGE Family Study. Metabolism 2000;49:1014-20. [PubMed: 10954019]

13. Meikle AW, Stephenson RA, Lewis CM, Wiebke GA, Middleton RG. Age, genetic, and nongenetic factors influencing variation in serum sex steroids and zonal volumes of the prostate and benign prostatic hyperplasia in twins. Prostate 1997;33:105-11. [PubMed: 9316651]

14. Jaquish CE, Blangero J, Haffner SM, Stern MP, MacCluer JW. Quantitative genetics of serum sex hormone-binding globulin levels in participants in the San Antonio Family Heart Study. Metabolism 1997;46:988-91. [PubMed: 9284884] 
15. Low YL, Dunning AM, Dowsett M, et al. Implications of gene-environment interaction in studies of gene variants in breast cancer: an example of dietary isoflavones and the D356N polymorphism in the sex hormone-binding globulin gene. Cancer Res 2006;66:8980-3. [PubMed: 16982738]

16. Dunning AM, Dowsett M, Healey CS, et al. Polymorphisms associated with circulating sex hormone levels in postmenopausal women. J Natl Cancer Inst 2004;96:936-45. [PubMed: 15199113]

17. Cousin P, Calemard-Michel L, Lejeune H, et al. Influence of SHBG gene pentanucleotide TAAAA repeat and D327N polymorphism on serum sex hormone-binding globulin concentration in hirsute women. J Clin Endocrinol Metab 2004;89:917-24. [PubMed: 14764814]

18. Haiman CA, Riley SE, Freedman ML, Setiawan VW, Conti DV, Le Marchand L. Common genetic variation in the sex steroid hormone-binding globulin (SHBG) gene and circulating shbg levels among postmenopausal women: the Multiethnic Cohort. J Clin Endocrinol Metab 2005;90:2198204. [PubMed: 15634719]

19. Eriksson AL, Lorentzon M, Mellström D, et al. SHBG gene promoter polymorphisms in men are associated with serum sex hormone-binding globulin, androgen and androgen metabolite levels, and hip bone mineral density. J Clin Endocrinol Metab 2006;91:5029-37. [PubMed: 16926255]

20. Zhao JL, Chen ZJ, Zhao YR, et al. Study on the (TAAAA)n repeat polymorphism in sex hormonebinding globulin gene and the SHBG serum levels in putative association with the glucose metabolic status of Chinese patients suffering from polycystic ovarian syndrome in Shandong province. Zhonghua Yi Xue Yi Chuan Xue Za Zhi 2005;22:644-7. [PubMed: 16331562](In Chinese.)

21. Cui Y, Shu XO, Cai Q, et al. Association of breast cancer risk with a common functional polymorphism (Asp327Asn) in the sex hormone-binding globulin gene. Cancer Epidemiol Biomarkers Prev 2005;14:1096-101. [PubMed: 15894658]

22. Berndt SI, Chatterjee N, Huang WY, et al. Variant in sex hormone-binding globulin gene and the risk of prostate cancer. Cancer Epidemiol Biomarkers Prev 2007;16:165-8. [PubMed: 17220347]

23. Liu S, Lee IM, Song Y, et al. Vitamin E and risk of type 2 diabetes in the Women's Health Study randomized controlled trial. Diabetes 2006;55:2856-62. [PubMed: 17003353]

24. Wacholder S, McLaughlin JK, Silverman DT, Mandel JS. Selection of controls in case-control studies. I. Principles. Am J Epidemiol 1992;135:1019-28. [PubMed: 1595688]

25. Sesso HD, Buring JE, Christen WG, et al. Vitamins E and C in the prevention of cardiovascular disease in men: the Physicians' Health Study II randomized controlled trial. JAMA 2008;300:212333. [PubMed: 18997197]

26. Reynders M, Anckaert E, Schiettecatte J, Smitz J. Evaluation of a new automated electrochemiluminescent sex hormone-binding globulin (SHBG) immunoassay. Clin Chem Lab Med 2005;43:86-9. [PubMed: 15653448]

27. Smith GD, Lawlor DA, Harbord R, Timpson N, Day I, Ebrahim S. Clustered environments and randomized genes: a fundamental distinction between conventional and genetic epidemiology. PLoS Med 2007;4(12):e352. [PubMed: 18076282]

28. Lawlor DA, Harbord RM, Sterne JA, Timpson N, Davey Smith G. Mendelian randomization: using genes as instruments for making causal inferences in epidemiology. Stat Med 2008;27:1133-63. [PubMed: 17886233]

29. Sheehan NA, Didelez V, Burton PR, Tobin MD. Mendelian randomisation and causal inference in observational epidemiology. PLoS Med 2008;5(8):e177. [PubMed: 18752343]

30. Thomas DC, Conti DV. Commentary: the concept of 'Mendelian Randomization'. Int J Epidemiol 2004;33:21-5. [PubMed: 15075141]

31. Hardin JW, Schmiediche H, Carroll RJ. Instrumental variables, bootstrapping, and generalized linear models. Stata J 2003;3:351-60.

32. Catalano MG, Frairia R, Boccuzzi G, Fortunati N. Sex hormone-binding globulin antagonizes the anti-apoptotic effect of estradiol in breast cancer cells. Mol Cell Endocrinol 2005;230:31-7. [PubMed: 15664449]

33. Bonds DE, Lasser N, Qi L, et al. The effect of conjugated equine oestrogen on diabetes incidence: the Women's Health Initiative randomised trial. Diabetologia 2006;49:459-68. [PubMed: 16440209]

34. Margolis KL, Bonds DE, Rodabough RJ, et al. Effect of oestrogen plus progestin on the incidence of diabetes in postmenopausal women: results from the Women's Health Initiative Hormone Trial. Diabetologia 2004;47:1175-87. [PubMed: 15252707] 
35. Ding EL, Song Y, Liu S. Testosterone, estradiol and type 2 diabetes. JAMA 2006;296:169-70. [PubMed: 16835421]

36. Vehkavaara S, Hakala-Ala-Pietilä T, Virkamäki A, et al. Differential effects of oral and transdermal estrogen replacement therapy on endothelial function in postmenopausal women. Circulation 2000;102:2687-93. [PubMed: 11094033]

37. Taskinen MR, Puolakka J, Pyörälä T, et al. Hormone replacement therapy lowers plasma Lp(a) concentrations: comparison of cyclic transdermal and continuous estrogen-progestin regimens. Arterioscler Thromb Vasc Biol 1996;16:1215-21. [PubMed: 8857916]

38. Selby P, McGarrigle HH, Peacock M. Comparison of the effects of oral and transdermal oestradiol administration on oestrogen metabolism, protein synthesis, gonadotrophin release, bone turnover and climacteric symptoms in postmenopausal women. Clin Endocrinol (Oxf) 1989;30:241-9. [PubMed: 2512035]

39. Haffner SM. Sex hormone-binding protein, hyperinsulinemia, insulin resistance and noninsulindependent diabetes. Horm Res 1996;45:233-7. [PubMed: 8964590]

40. Kalme T, Koistinen H, Loukovaara M, Koistinen R, Leinonen P. Comparative studies on the regulation of insulin-like growth factor-binding protein-1 (IGFBP-1) and sex hormone-binding globulin (SHBG) production by insulin and insulin-like growth factors in human hepatoma cells. J Steroid Biochem Mol Biol 2003;86:197-200. [PubMed: 14568572] 


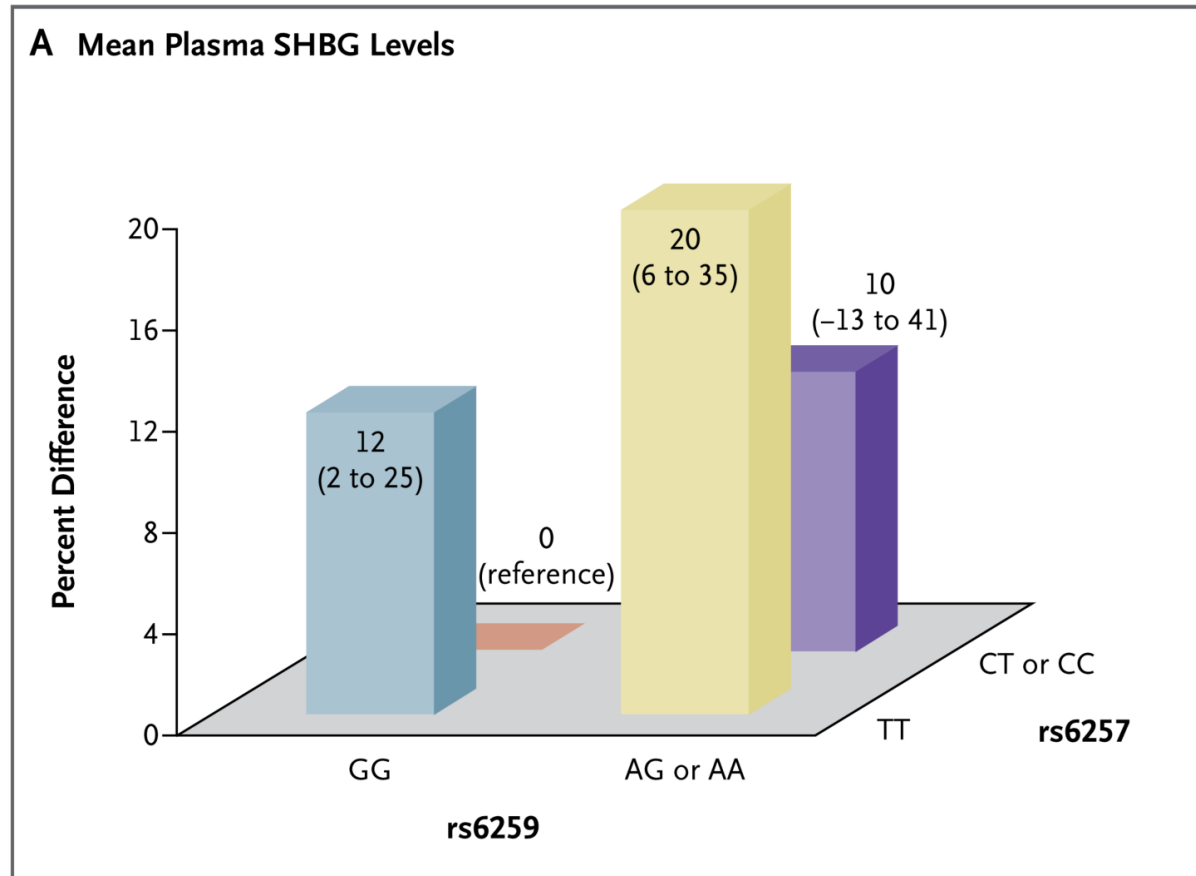

B Risk of Type 2 Diabetes

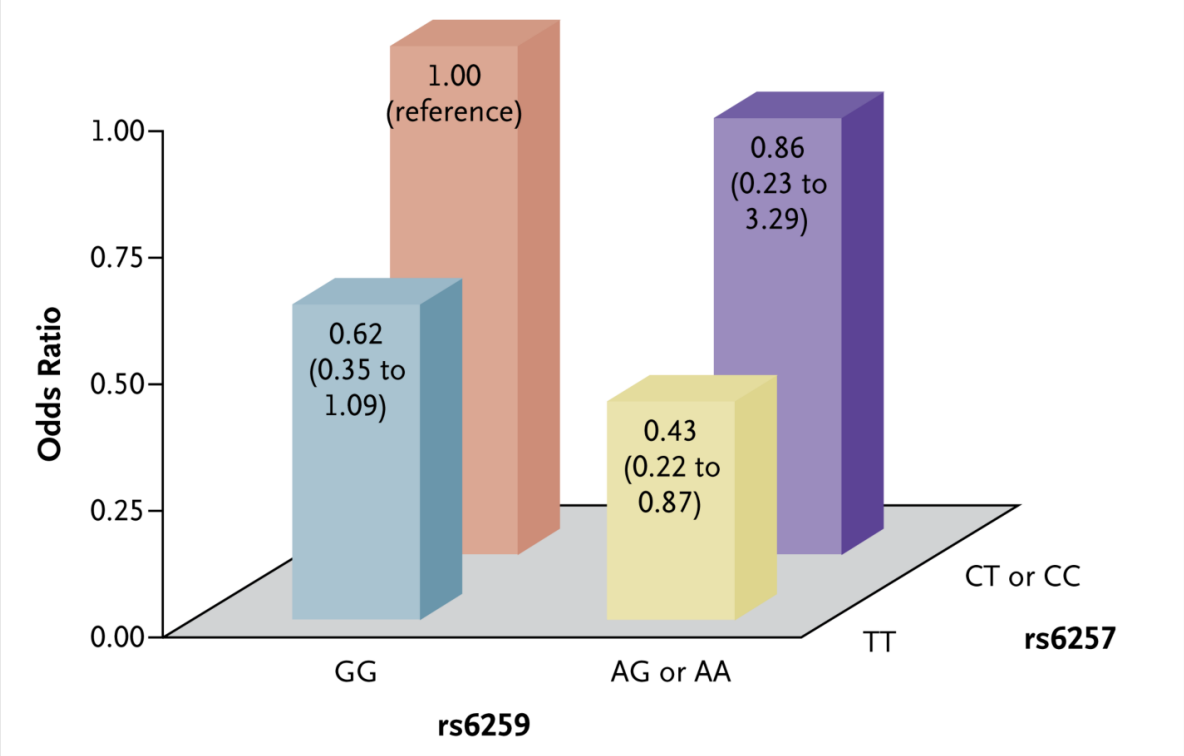

Figure 1. Plasma Levels of Sex Hormone-Binding Globulin (SHBG) and Risk of Type 2 Diabetes in Women, According to $S H B G$ Genotypes

Panel A shows the percent changes in SHBG levels for each of three variant-genotype groups as compared with carriers of the rs6257 variant allele, who were also homozygous for the rs6259 wild-type allele (associated with the lowest SHBG level). Panel B shows the odds of type 2 diabetes among the same genotype groups. The $95 \%$ confidence intervals are given in parentheses. 
Table 1

Baseline Characteristics of Case Patients and Controls. ${ }^{*}$

\begin{tabular}{|c|c|c|c|}
\hline $\begin{array}{l}\text { Characteristic } \\
\text { Women }\end{array}$ & Case Patients & Controls & P Value ${ }^{t}$ \\
\hline No. of participants & 359 & 359 & \\
\hline Age - yr & $60.3 \pm 6.1$ & $60.3 \pm 6.1$ & \\
\hline White race $-\%^{\ddagger}$ & 92.5 & 92.5 & \\
\hline Body-mass index $x^{\S}$ & $30.9 \pm 6.1$ & $26.0 \pm 4.9$ & $<0.001$ \\
\hline Alcohol use - g/day & $2.62 \pm 7.4$ & $4.19 \pm 8.3$ & 0.007 \\
\hline Current smoking - \% & 14.2 & 13.7 & 0.83 \\
\hline Strenuous physical activity $\geq$ once/wk $-\%$ & 30.7 & 38.7 & 0.06 \\
\hline Past postmenopausal hormone use - $\%$ & 32.0 & 27.9 & 0.22 \\
\hline Any oral contraceptive use - \% & 49.9 & 47.1 & 0.23 \\
\hline Age at menopause - yr & $48.0 \pm 6.1$ & $48.0 \pm 5.7$ & 0.92 \\
\hline Time since menopause - yr & $12.3 \pm 8.2$ & $12.1 \pm 7.9$ & 0.88 \\
\hline Natural cause of menopause - \% & 63.0 & 69.4 & 0.08 \\
\hline Age at menarche, $<12 \mathrm{yr}-\%$ & 25.4 & 21.7 & 0.46 \\
\hline Age at first pregnancy of $\geq 6$-mo gestation, $<25 \mathrm{yr}-\%$ & 56.8 & 48.8 & 0.05 \\
\hline Family history of diabetes - $\%$ & 48.5 & 24.0 & $<0.001$ \\
\hline History of hypertension - $\%$ & 50.1 & 30.4 & $<0.001$ \\
\hline$\geq 5$ Pregnancies $-\%$ & 30.1 & 34.0 & 0.10 \\
\hline Currently married - \% & 63.5 & 65.2 & 0.15 \\
\hline College graduate - \% & 30.4 & 38.7 & 0.05 \\
\hline SHBG - nmol/liter & $22.3 \pm 13.8$ & $36.9 \pm 17.4$ & $<0.001$ \\
\hline \multicolumn{4}{|l|}{ SHBG SNP - no./total no. with data (\%) } \\
\hline rs6257 & & & 0.08 \\
\hline Variant allele $\mathrm{C}$ & $76 / 337(22.6)$ & 65/344 (18.9) & \\
\hline Wild-type allele T & $261 / 337(77.4) 2$ & $79 / 344(81.1)$ & \\
\hline rs 6258 & & & 0.91 \\
\hline Variant allele T & 2/339 (0.6) & 4/347 (1.2) & \\
\hline Wild-type allele C & $337 / 339(99.4) 3$ & $43 / 347(98.8)$ & \\
\hline rs6259 & & & 0.33 \\
\hline Variant allele A & $66 / 342(19.3)$ & $82 / 337(24.3)$ & \\
\hline Wild-type allele G & $276 / 342(80.7) 2$ & $55 / 337(75.7)$ & \\
\hline \multicolumn{4}{|l|}{ Men } \\
\hline No. of participants & 170 & 170 & \\
\hline Age - yr & $63.7 \pm 7.6$ & $63.7 \pm 7.6$ & \\
\hline White race $-\%^{*}$ & 85.3 & 85.3 & \\
\hline Body-mass index ${ }^{\S}$ & $28.9 \pm 3.9$ & $25.5 \pm 3.4$ & $<0.001$ \\
\hline Alcohol use of $\geq 1$ drink/wk $-\%$ & 61.2 & 62.4 & 0.16 \\
\hline Current smoking - \% & 6.5 & 1.2 & 0.06 \\
\hline Vigorous physical activity $\geq 1$ day/wk $-\%$ & 55.3 & 65.9 & 0.34 \\
\hline Current multivitamin use - $\%$ & 27.7 & 27.1 & 0.90 \\
\hline Systolic blood pressure $-\mathrm{mm} \mathrm{Hg}$ & $133 \pm 14$ & $127 \pm 11$ & $<0.001$ \\
\hline Hyperlipidemia - \% & 67.1 & 58.8 & 0.09 \\
\hline Family history of diabetes - \% & 32.9 & 17.1 & 0.05 \\
\hline SHBG - nmol/liter & $19.6 \pm 7.2$ & $27.3 \pm 10.7$ & $<0.001$ \\
\hline \multicolumn{4}{|l|}{ SHBG SNP - no./total no. with data (\%) } \\
\hline rs6257 & & & 0.64 \\
\hline Variant allele $\mathrm{C}$ & $33 / 167(19.8)$ & $29 / 164(17.7)$ & \\
\hline Wild-type allele T & $134 / 167(80.2) 1$ & $35 / 164(82.3)$ & \\
\hline rs 6259 & & & 0.98 \\
\hline Variant allele A & $27 / 167(16.2)$ & $41 / 163(25.2)$ & \\
\hline Wild-type allele G & $140 / 167(83.8) 1$ & $22 / 163(74.8)$ & \\
\hline
\end{tabular}

Plus-minus values are means \pm SD. SHBG denotes sex hormone-binding globulin, and SNP single-nucleotide polymorphism.

${ }^{\dagger} \mathrm{P}$ values for continuous variables were calculated by means of mixed-effects models used to determine the mean difference between case patients and controls; for categorical variables, by means of tests for homogeneity across levels, from conditional logistic-regression analysis; and for SNPs, by means of tests of Hardy-Weinberg equilibrium among controls. P values are not shown for the variables used to match case patients and controls (age and race).

${ }^{\dagger}$ Race was self-reported.

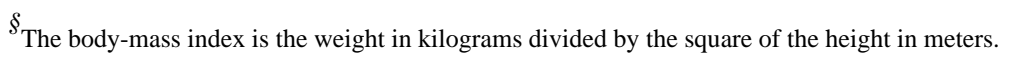




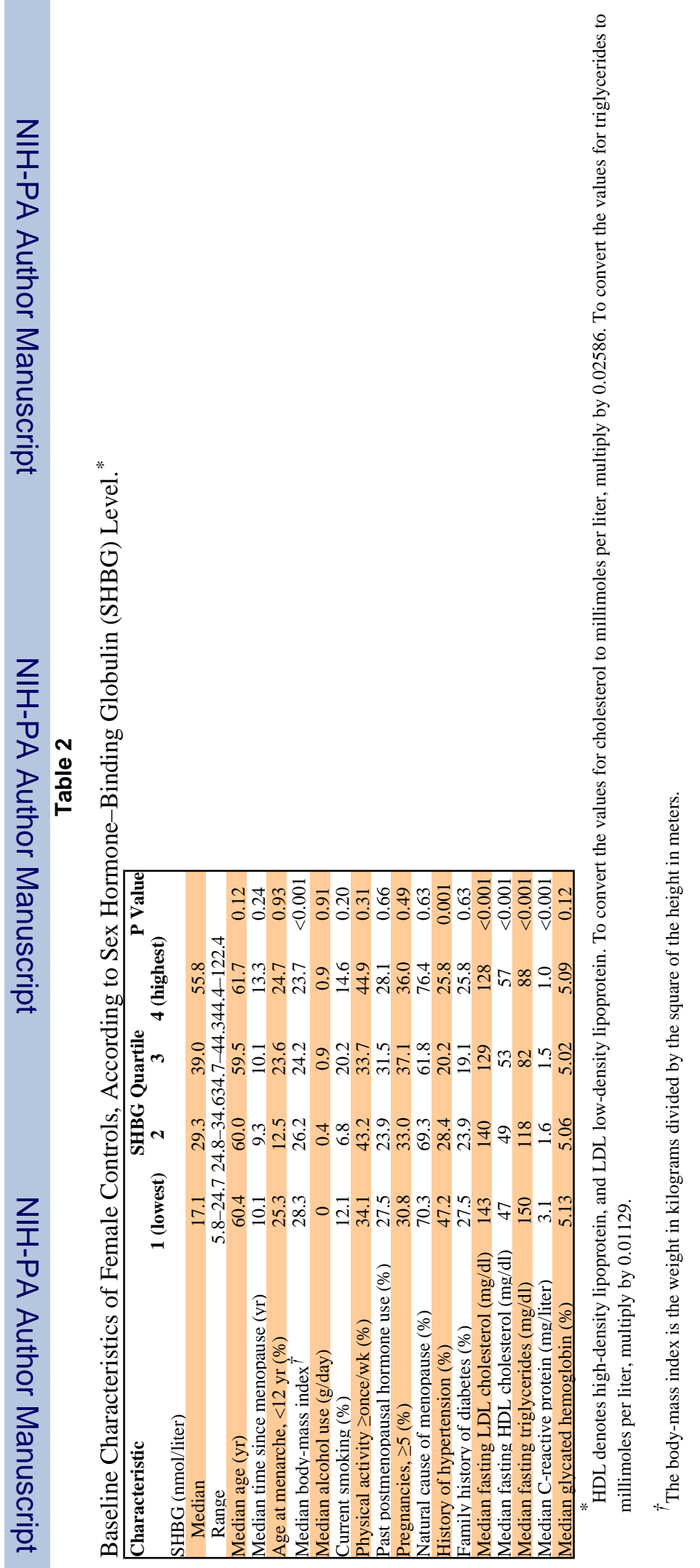

N Engl J Med. Author manuscript; available in PMC 2010 September 17. 


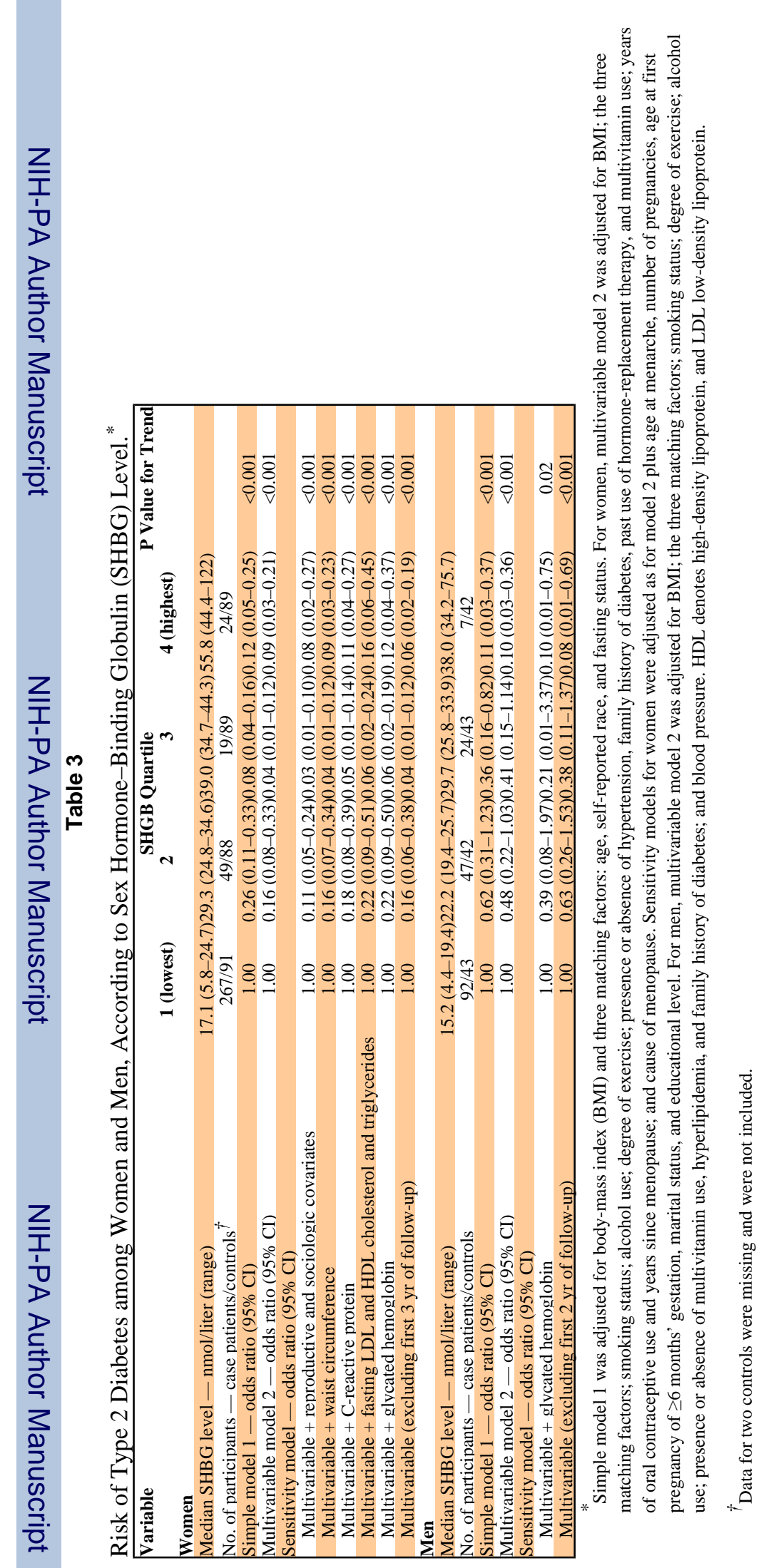

N Engl J Med. Author manuscript; available in PMC 2010 September 17. 


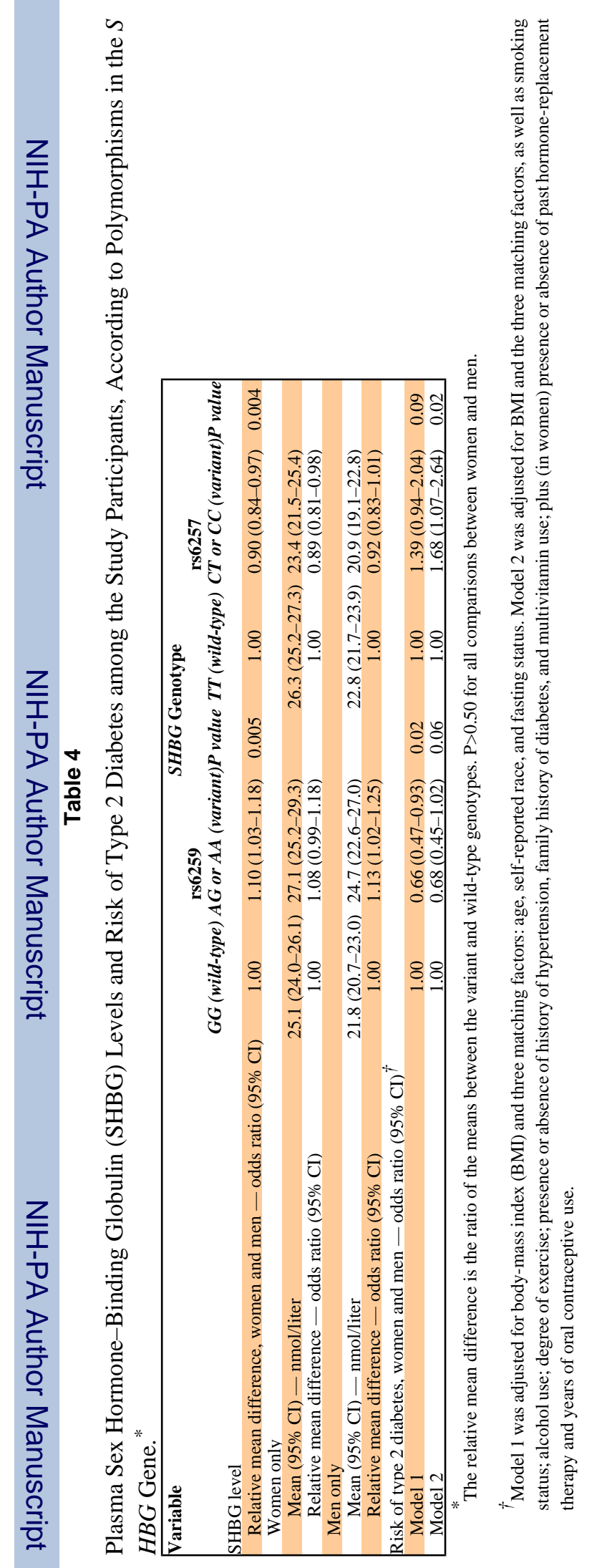

N Engl J Med. Author manuscript; available in PMC 2010 September 17. 


\section{Table 5}

Odds Ratios for Type 2 Diabetes per Unit of Increase in the Sex Hormone-Binding Globulin (SHBG) Level. ${ }^{*}$ Analysis Odds Ratio (95\% CI)

Mendelian randomization analysis ${ }^{\dagger}$

Allele rs6259 and rs6257

Women

$0.28(0.13-0.58)$

Allele rs6259 $0.29(0.15-0.58)$

Women and men $\quad 0.23(0.10-0.54)$

Women only $\quad 0.19(0.04-1.01)$

Men only $\quad 0.25(0.09-0.65)$

Allele rs6257

Women and men $\quad 0.40(0.19-0.88)$

Women only $\quad 0.39(0.17-0.88)$

Men only $\S \quad 0.53(0.05-5.22)$

Conventional multivariable analysis ${ }^{I}$

Women

Simple model $1 \quad 0.40(0.31-0.51)$

Multivariable model $\quad 0.34(0.26-0.45)$

Men

Simple model $1 \quad 0.43(0.31-0.59)$

Multivariable model $\quad 0.39(0.27-0.58)$

The unit of increase measured was the natural-log standard-deviation in sex-specific control distributions.

The mendelian randomization analyses involved $S H B G$ genotypes as instrumental variables in multivariable generalized linear models. 31

${ }^{*} \mathrm{P}=0.78$ and $\mathrm{P}=0.81$ for the difference between men and women in carriage of a variant rs6259 allele and carriage of a variant rs6257 allele, respectively. All $\mathrm{P}=0.001$ for mendelian instrument rs6259 and for joint analyses. $\mathrm{P}=0.02$ for mendelian instrument $\mathrm{rs} 6257$, and $\mathrm{P}<0.001$ for the conventional multivariable analyses.

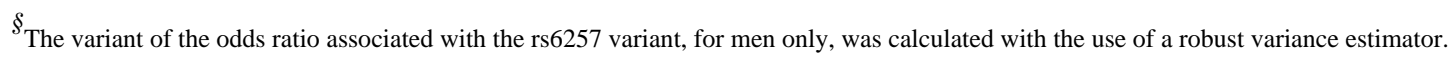

${ }^{I}$ Conventional models were adjusted for covariates as described for each model in Table 3. 